\title{
Hub genes and key pathways of non-small lung cancer identified using bioinformatics
}

\author{
QING TANG $^{1^{*}}, \mathrm{HONGMEI} \mathrm{ZHANG}^{2^{*}}, \mathrm{MAN} \mathrm{KONG}^{2}$, XIAOLI MAO ${ }^{2}$ and XIAOCUI CAO ${ }^{2}$ \\ ${ }^{1}$ Department of Clinical Laboratory, Tongji Hospital; ${ }^{2}$ Department of Clinical Laboratory, The Central Hospital of Wuhan, \\ Tongji Medical College, Huazhong University of Science and Technology, Wuhan, Hubei 430014, P.R. China
}

Received February 13, 2017; Accepted February 5, 2018

DOI: $10.3892 / \mathrm{ol} .2018 .8882$

\begin{abstract}
Non-small cell lung cancer (NSCLC) is the most common type of lung cancer, accounting for $\sim 80 \%$ of all lung cancer cases. The aim of the present study was to identify key genes and pathways in NSCLC, in order to improve understanding of the mechanism of lung cancer. The GSE33532 gene expression dataset, containing 20 normal and 80 NSCLC samples, was used. Gene Ontology (GO) and Kyoto Encyclopedia of Genes and Genomes (KEGG) analyses were performed to obtain the enrichment data of differently expressed genes (DEGs). Disease modules within NSCLC were constructed by Cytoscape, using protein-protein interaction (PPI) from the Search Tool for the Retrieval of Interacting Genes database. In addition, the Kaplan Meier plotter KMplot was used to assess the top hub genes in the PPI network. As a result, 1,795 genes were identified in NSCLC; 729 were upregulated and 1,066 were downregulated. The results of the GO analysis indicated that the upregulated DEGs were significantly enriched in 'biological processes' (BP), including 'cell cycle and nuclear division'; the downregulated DEGs were also significantly enriched in $\mathrm{BP}$, including 'response to wounding', 'anatomical structure morphogenesis' and 'response to stimulus'. Upregulated DEGs were also enriched in 'cell cycle', 'DNA replication' and the 'tumor protein 53 signaling pathway', while the downregulated DEGs were also enriched in 'complement and coagulation cascades', 'malaria' and 'cell adhesion molecules'. The top 9 hub genes were cyclin-dependent kinase 9 (CDK1), polo-like kinase 1 , aurora kinase B, cell division cycle 20, baculoviral initiator of apoptosis repeat containing 5, mitotic checkpoint serine/threonine kinase $\mathrm{B}$, proliferating cell nuclear antigen
\end{abstract}

Correspondence to: Professor Xiaocui Cao, Department of Clinical Laboratory, The Central Hospital of Wuhan, Tongji Medical College, Huazhong University of Science and Technology, 26 Shengli Road, Wuhan, Hubei 430014, P.R. China

E-mail: caoxiaocuizxh@126.com

*Contributed equally

Key words: non-small cell lung cancer, microarray, pathway, enrichment, different expression
(PCNA), centromere protein A and MAD2 mitotic arrest deficient-like 1, and the KMplot results revealed that the high expression levels of these genes resulted in significantly low survival rates, compared with low expression samples $(\mathrm{P}<0.05)$, with the exception of PCNA and CDK1. In the pathway crosstalk analysis, 26 nodes and 41 interactions were divided into two groups: One module of the two groups primarily included 'metabolism of amino acid' and the other primarily contained 'tumor necrosis signaling' pathways. In conclusion, the present study assisted in improving the understanding of the molecular mechanisms underlying NSCLC development, and the results may help the understanding of the biological mechanism of NSCLC.

\section{Introduction}

Lung cancer is the leading cause of cancer-associated mortality worldwide, accounting for 1.3 million mortalities annually (World Health Organization, 2008) (1). It has the highest mortality rate of all types of cancer in women and men globally, with its mortality rate exceeding the combined mortality rates of breast, prostate, colorectal and pancreatic cancer (1). Non-small cell lung cancer (NSCLC) accounts for $\sim 80 \%$ of all lung cancer cases globally, with $\sim 75 \%$ of patients being diagnosed in the middle-late stages, and the 5-year survival rate of NSCLC is poor (mean, 9-11 months) (2). NSCLC includes squamous cell carcinoma, adenocarcinoma and large cell carcinoma subtyes (3); and NSCLC cells divide more slowly and spread relatively late compared with small-cell carcinoma cells. At present, the lack of knowledge concerning the molecular mechanisms of NSCLC progression has limited the development of novel treatment strategies. However, in combination with a large number of applications involving bioinformatics available for clinical studies, a large volume of disease-associated bioinformatics data has been produced. Obtaining detailed biological information from these resources is valuable for the study and development of therapeutic strategies for NSCLC.

High-throughput bioinformatics platforms may promote the analysis of differential gene expression, including microarrays, and have a wide range of applications in medical oncology, particularly in searching for disease-associated biomarkers (4), alternative splicing (5) and gene function prediction (6). Numerous previous studies have generated a 
large volume of microarray data, and a number of gene expression profiling studies on NSCLC have identified differentially expressed genes (DEGs) in various pathways, molecular functions and biological processes.

Therefore, in the present study, the original data (GSE33532) was downloaded from the Gene Expression Omnibus (GEO; http://www.ncbi.nlm.nih.gov/geo/), which contains variations in gene expression profiles in stage I and II (Tumor-Node-Metastasis classification of malignant tumors) (7) NSCLC tissues (8), and in normal tissues. The gene expression profiling of NSCLC tissues has resulted in the establishment of several prognostic and predictive gene signatures with little overlap (8). Subsequently, R software (version 3.4.1; https://www.r-project.org/) was used to compare the expression profiles of NSCLC tissues with those of normal tissues in order to identify DEGs. Subsequent to obtaining the DEGs, biological function enrichment and integrated protein-protein interaction network (PPI) analyses were performed to establish the complete characterization of the DEGS for NSCLC and obtain further understanding into the mechanism underlying NSCLC. By analyzing the biological function of the DEGs, certain potential biomarkers were identified for additional study.

\section{Materials and methods}

Microarray data. The GSE33532 microarray expression dataset was downloaded from the GEO database (http://www.ncbi. nlm.nih.gov/geo/). This dataset was based on the Affymetrix GPL570 platform (Affymetrix Human Genome U133 Plus 2.0 Array), submitted by Meister et al (8). The GSE33532 dataset contained 100 samples, including 80 NSCLC tissue samples and 20 normal tissue samples.

Identification of DEGs. The raw data used for analysis contained CEL files (GPL570 platform), and the results were obtained using Affy package (version 1.52.0) (9), Limma package (version 3.0.1) (10) and Gplot package (version 3.3.2; https://cran.r-project.org/web/packages/gplots/). A hierarchical clustering method was applied to classify these data into either the NSCLC or normal group. The quality control was indicated by using the 'Robust Multiarray Averaging' (RMA) function in the Affy packages. Subsequently, the adjust method 'BH' in the Limma $\mathrm{R}$ package was used to identify DEGs with log Ifold changel $>1$ and adj. $\mathrm{P}<0.05$ as cut-off levels for statistically significant candidate genes. Following this, a heatmap was constructed to indicate the differential expression levels of the top 100 DEGs (50 upregulated and 50 downregulated), and a volcano plot was produced to map all DEGs in this dataset.

Gene Ontology (GO) and kyoto encyclopedia of genes and genomes (KEGG) analyses. In the field of molecular biology, GO is the most developed and widely-used ontology. Through the GO method, it is also possible to characterize biological concepts with different specificity levels, from general to precise concepts (11). KEGG (http://www.genome.ad.jp/kegg/) is a collection of databases and associated software for understanding and simulating higher-order functional behaviors of cells or organisms from their genomic information (12). In addition, analyzing DEGs using the Database for Annotation, Visualization and Integrated Discovery (DAVID; http://david. ncifcrf.gov/) is an important means of identifying the relevant biological functions for any high-throughput gene functional analysis (13). The DEGs of the present study were analyzed to identify their biological function using the results from the GO and KEGG pathway analyses and the DAVID online tool. $\mathrm{P}<0.05$ was considered to indicate a statistically significant difference.

Disease module creation using the integration of PPIs. The Search Tool for the Retrieval of Interacting Genes (STRING) database includes 5,214,234 proteins from 1,133 organisms. It identified the PPIs of the DEGs identified in the present study. To evaluate their interactive associations, all DEGs were mapped to this database, in order to get an improved result, interactions with the highest confidence score (score $>0.9$ ) in the STRING database were selected. Subsequently, the PPIs were analyzed by Cytoscape software (version 3.2.1; National Resource for Network Biology) (14) to obtain the PPI network. The criteria of disease module searching were set as follows: Molecular COmplex DEtection (MCODE) score $>3$, and each module must have $>4$ nodes. $\mathrm{P}<0.05$ was considered to indicate a statistically significant difference.

Pathway crosstalk. The regulation of biological pathways is complex, yet it underlies the functional coordination of cells (15). Cancer is a disease that is characterized by unregulated cell proliferation, driven by underlying pathway deregulation (16). This pathway deregulation occurs within and between pathways (17). Pathway crosstalk analysis may assist in identifying the interactions among pathways enriched by DEGs. The present study used the pathway information of DEGs from the KEGG database to conduct a pathway crosstalk analysis in NSCLC. The principle of pathway crosstalk is defined by $>3$ overlapping genes in 2 pathways (each pathway must have $\leq 5$ genes) (17). To measure the interaction of crosstalk, 2 novel variables were introduced: The Jaccard Coefficient (JC) ${ }^{\mathrm{JC}}-\frac{A^{A} \cap^{B}}{A \backslash \mathrm{B}}$ and the Overlap Coefficient (OC)

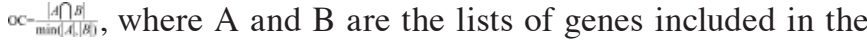
2 analyzed pathways. The rank value (RV) was calculated by $\frac{I C+O C}{2}(17)$. Subsequently, Cytoscape software was used to map the interaction between pathways and use RV as the interaction type in order to display the weight of the crosstalk.

Survival analysis. The Kaplan-Meier plotter (KMplot, http://www.kmplot.com/analysis) is capable of assessing the effect of 54,675 genes on survival using 10,293 cancer samples. These include 5,143 breast, 1,648 ovarian, 2,437 lung and 1,065 gastric cancer samples, with mean follow-up periods of $69,40,49$ and 33 months, respectively. The primary purpose of this tool is to conduct meta-analysis-based biomarker assessments (18). The top 9 hub genes of disease module in the present study were entered into the KMplot database to examine the association between these genes and the 5-year survival rates of patients.

\section{Results}

Identification of DEGs. The dataset of the present study contained 100 samples; 20 normal tissue samples and 80 NSCLC tissue samples. Each sample from the chip was 

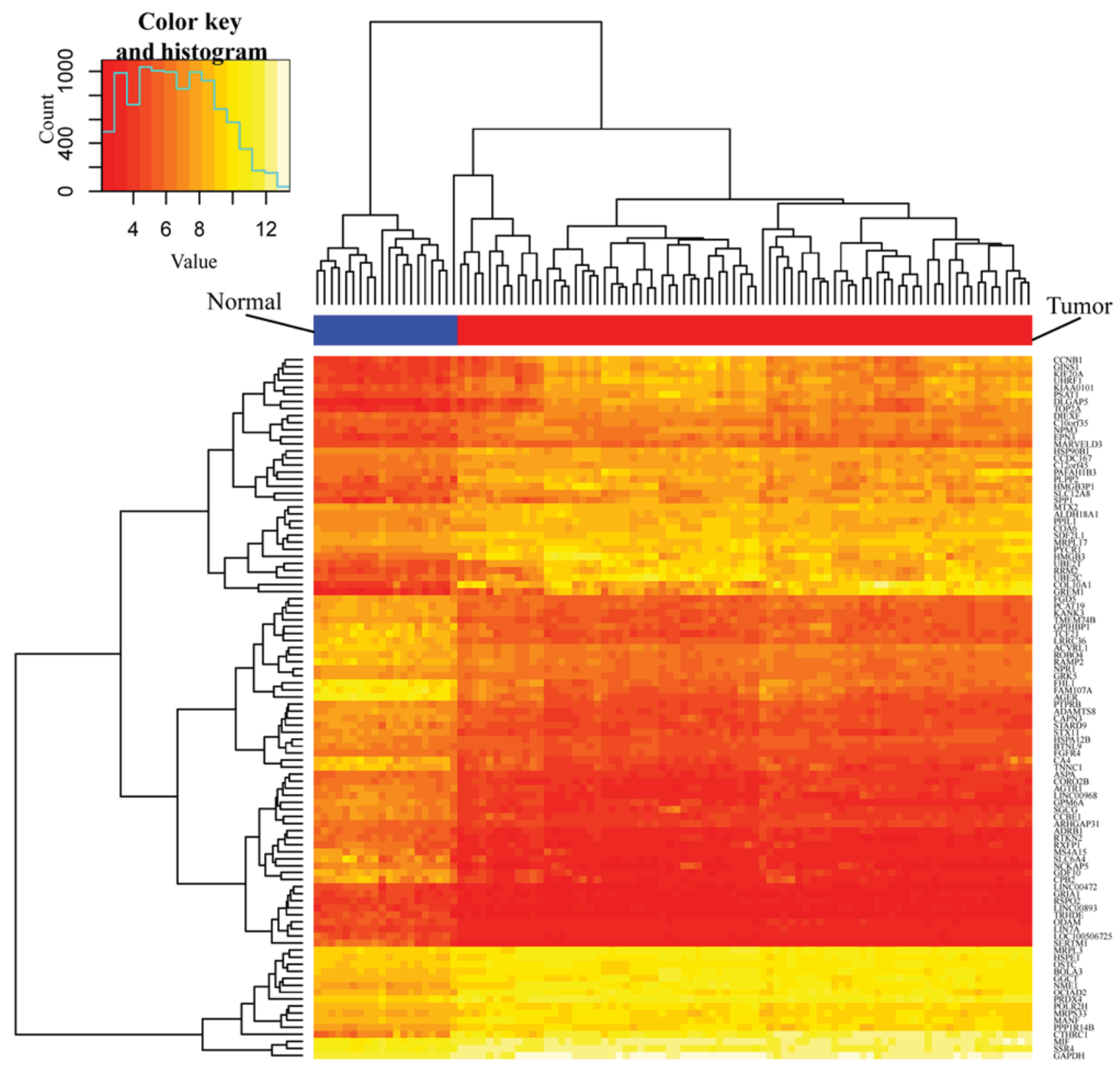

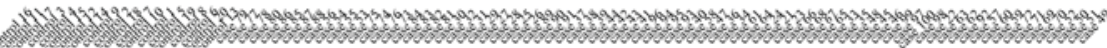

Figure 1. Heat map of the top 100 differentially expressed genes (top 50 upregulated and top 50 downregulated genes). Yellow denotes upregulation and red represents downregulation. The blue and red bar represents control and case group, respectively.

analyzed by Affy and Limma packages of R software, respectively. Based on the Affy package, using the RMA method to pre-process the dataset, then using the lmFit function of the Limma package to screen differentially expressed genes with $\mathrm{P}<0.05$ and fold control $(\log \mid \mathrm{FCl})>1$ criteria to obtain DEGs from the dataset, a total of 1,795 genes were identified. Of these, 729 were upregulated and 1,066 were downregulated. The Gplots package (R software) was used to obtain the top 50 upregulated and the top 50 downregulated DEGs, and to produce an expression heatmap (Fig. 1) and a volcano plot of the DEG distribution (Fig. 2).

GO enrichment analysis. Enriched GO categories and KEGG pathways were identified by uploading all DEGs to DAVID. The results of the GO analysis indicated that the upregulated DEGs were significantly enriched in 'biological processes' (BP), which included 'cell cycle' and 'nuclear division' (Table I); downregulated DEGs were also significantly enriched in BP, including 'response to wounding', 'anatomical structure morphogenesis' and 'response to stimulus' (Table I). For 'molecular function', the upregulated DEGs were enriched in 'microtubule motor activity', 'protein binding' and 'structural molecule activity', and the downregulated DEGs were enriched in 'calcium ion binding', 'protein binding' and 'growth factor binding' (Table I). Concurrently, the GO 'cell component' analysis also revealed that the upregulated DEGs were significantly enriched in 'chromosome' and 'centromeric region', and that the downregulated DEGs were enriched in 'plasma membrane part', 'extracellular region part' and 'cell periphery' (Table I). 


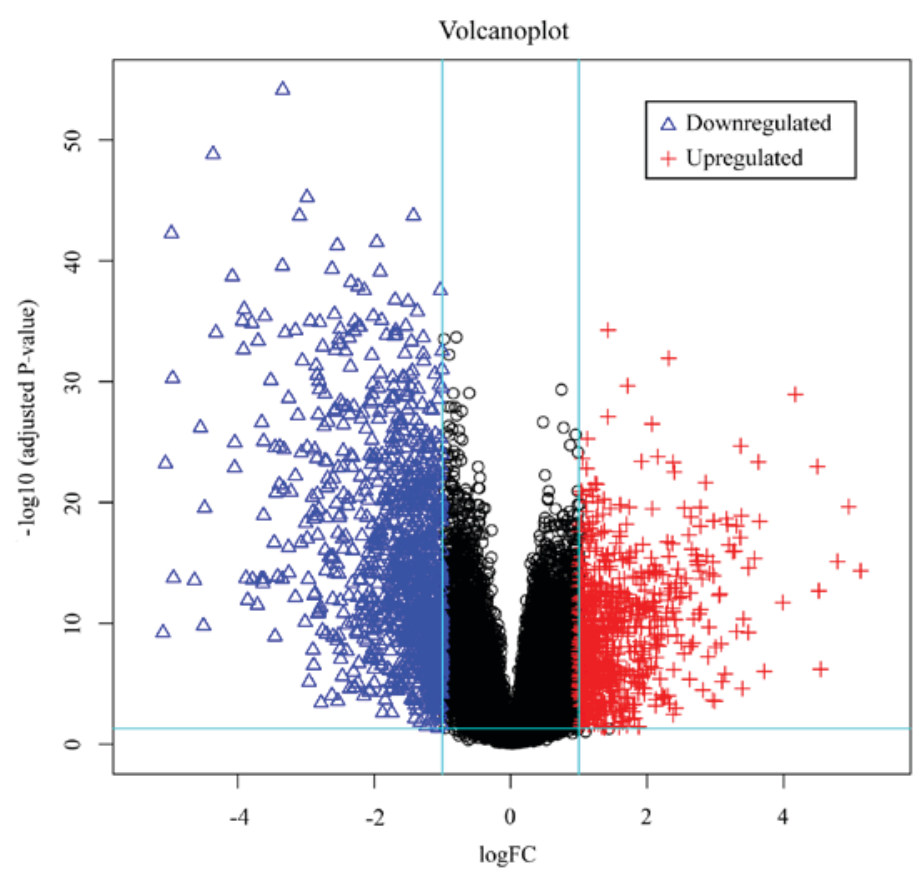

Figure 2. Volcano plot of the distribution of all differentially expressed genes, mapping the 729 upregulated genes (red crosses) and 1,066 downregulated genes (blue triangles). FC, fold change.

KEGG pathway analysis. Table II indicates the KEGG analysis result of the most significantly enriched pathways (top 5 upregulated and downregulated) in the DEGs of NSCLC. The upregulated DEGs were significantly enriched in 'cell cycle', 'DNA replication', 'tumor protein 53 (p53) signaling pathway', 'Extracellular matrix (ECM)-receptor interaction' and 'Protein digestion and absorption', while the downregulated DEGs were significantly enriched in 'Complement and coagulation cascades', 'Malaria', 'Cell adhesion molecules (CAMs)', 'Axon guidance' and 'Renin secretion'.

Integration of PPIs create disease module. All DEGs of NSCLC were loaded into the STRING database, to obtain the PPI data among them, and PPIs with highest interaction score (confidence $>0.9$ ) were selected. Subsequently, Cytoscape was used to identify the 9 hub nodes with the highest degrees, which included aurora kinase B (AURKB), centromere protein A (CENPA), cyclin dependent kinase 1 (CDK1), proliferating cell nuclear antigen (PCNA), BUB1 mitotic checkpoint serine/threonine kinase B (BUB1B), cell division cycle 20 (CDC20), baculoviral Inhibitor of apoptosis (IAP) repeat containing 5 (BIRC5), MAD2 mitotic arrest deficient-like 1 (MAD2L1) and polo like kinase 1 (PLK1). Of these hub genes (Table III), CDK1 revealed the highest node degree (degree $=58$ ). In addition, a total of 684 nodes and 2,134 edges were analyzed using MCODE in Cytoscape software. The top 5 largest size modules were identified, and the functional annotations of the genes within them were isolated (Fig. 3). KEGG enrichment analysis of these modules demonstrated that the genes in modules 1-5 were primarily associated with 'cell cycle', 'chemokine signaling pathway', 'protein digestion and absorption', 'DNA replication' and 'malaria'.

Pathwaycrosstalk.Inordertoidentify the significantly-enriched pathways and to understand the interaction between them, pathway crosstalk analysis among the 26 significant pathways was performed; in total, these pathways contained 41 edges. Based on the crosstalk analysis, the pathways were divided into two major groups, and each of the included pathways shared more crosstalk events than those outside of the pathways identified in the crosstalk analysis and may be associated with similar biological processes (Fig. 4). One group primarily included metabolism of amino acids tyrosine, phenylalanine, tryptophan and histidine. The other group primarily contained tumor necrosis signaling pathways: CAM; tumor necrosis factor signaling pathway; leukocyte transendothelial migration; malaria and stress reaction (renin secretion, salivary secretion, platelet activation, vascular smooth muscle contraction, extracellular matrix (ECM)-receptor interaction and oocyte meiosis). These pathways had $>4$ degrees and high rank-values with other pathways in this crosstalk.

Survival analysis. To validate the 9 hub genes identified, the KMplot was used to analyze the survival potential of patients with upregulated hub genes. Following the gene upload, 8 genes were available in KMplot database, and there were 1,926 patients as candidates. All the hub genes were upregulated genes, 7 of which were a significantly associated with low survival rates $(\mathrm{P}<0.05$; Fig. 5). However, PCNA was upregulated, but did not exhibit a significant association.

\section{Discussion}

NSCLC accounts for $\sim 80 \%$ of all types of diagnosed lung cancer (19). The cause of NSCLC is complex with various factors, including smoking, air pollution and radon exposure (20); therefore, understanding the biological mechanisms of NSCLC is important for clinical diagnosis and treatment. As microarrays have a wide range of applications in oncology, including identification of 
Table I. Gene Ontology analysis of DEGs associated with non-small cell lung cancer.

A, Upregulated DEGs

\begin{tabular}{|c|c|c|c|}
\hline Category & Term/gene function & Gene count & P-value \\
\hline $\mathrm{BP}$ & GO:0022403; cell cycle phase & 117 & $3.56 \times 10^{-30}$ \\
\hline $\mathrm{BP}$ & GO:0000278; mitotic cell cycle & 106 & $3.67 \times 10^{-27}$ \\
\hline $\mathrm{BP}$ & GO:0022402; cell cycle process & 124 & $3.65 \times 10^{-26}$ \\
\hline $\mathrm{BP}$ & GO:0000087; M phase of mitotic cell cycle & 71 & $4.22 \times 10^{-26}$ \\
\hline $\mathrm{BP}$ & GO:0000280; nuclear division & 69 & $1.79 \times 10^{-25}$ \\
\hline $\mathrm{MF}$ & GO:0003777; microtubule motor activity & 13 & $4.16 \times 10^{-6}$ \\
\hline MF & GO:0005515; protein binding & 323 & $1.48 \times 10^{-5}$ \\
\hline MF & GO:0005198; structural molecule activity & 45 & $1.59 \times 10^{-5}$ \\
\hline MF & GO:0016538; cyclin-dependent protein kinase regulator activity & 6 & $4.69 \times 10^{-5}$ \\
\hline MF & GO:0003678; DNA helicase activity & 8 & $2.00 \times 10^{-4}$ \\
\hline $\mathrm{CC}$ & GO:0044427; chromosomal part & 64 & $2.90 \times 10^{-17}$ \\
\hline $\mathrm{CC}$ & GO:0005694; chromosome & 70 & $5.28 \times 10^{-17}$ \\
\hline $\mathrm{CC}$ & GO:0000793; condensed chromosome & 34 & $2.21 \times 10^{-16}$ \\
\hline $\mathrm{CC}$ & O:0000775; chromosome, centromeric region & 32 & $2.73 \times 10^{-16}$ \\
\hline $\mathrm{CC}$ & GO:0000779; condensed chromosome, centromeric region & 25 & $5.44 \times 10^{-16}$ \\
\hline
\end{tabular}

B, Downregulated DEGs

\begin{tabular}{|c|c|c|c|}
\hline Category & Term/gene function & Gene count & P-value \\
\hline $\mathrm{BP}$ & GO:0009611; response to wounding & 130 & $1.29 \times 10^{-18}$ \\
\hline $\mathrm{BP}$ & GO:0009653; anatomical structure morphogenesis & 198 & $1.74 \times 10^{-18}$ \\
\hline $\mathrm{BP}$ & GO:0050896; response to stimulus & 468 & $3.25 \times 10^{-18}$ \\
\hline $\mathrm{BP}$ & GO:0042221; response to chemical stimulus & 239 & $5.30 \times 10^{-17}$ \\
\hline BP & GO:0044707; single-multicellular organism process & 406 & $2.16 \times 10^{-16}$ \\
\hline MF & GO:0005509; calcium ion binding & 65 & $1.64 \times 10^{-7}$ \\
\hline $\mathrm{MF}$ & GO:0005515; protein binding & 434 & $2.35 \times 10^{-7}$ \\
\hline MF & GO:0019838; growth factor binding & 20 & $2.36 \times 10^{-7}$ \\
\hline MF & GO:0005102; receptor binding & 99 & $5.28 \times 10^{-7}$ \\
\hline MF & GO:0097367; carbohydrate derivative binding & 27 & $7.64 \times 10^{-7}$ \\
\hline $\mathrm{CC}$ & GO:0044459; plasma membrane part & 200 & $2.10 \times 10^{-24}$ \\
\hline $\mathrm{CC}$ & GO:0044421; extracellular region part & 139 & $2.28 \times 10^{-24}$ \\
\hline $\mathrm{CC}$ & GO:0071944; cell periphery & 346 & $2.98 \times 10^{-21}$ \\
\hline $\mathrm{CC}$ & GO:0005886; plasma membrane & 338 & $2.20 \times 10^{-20}$ \\
\hline $\mathrm{CC}$ & GO:0005615; extracellular space & 105 & $1.81 \times 10^{-17}$ \\
\hline
\end{tabular}

DEGs, differentially expressed genes; BP, biological process; MF, molecular function; CC, cellular component.

disease-associated biomarkers, alternative splicing and gene function prediction, microarray data were extracted from GSE33532, and 729 upregulated and 1,066 downregulated DEGs between NSCLC and normal samples were identified using bioinformatics analysis. In order to obtain additional analysis of these DEGs, GO and KEGG analyses were performed using DAVID software.

The GO analysis results indicated that the upregulated DEGs were primarily associated with 'cell cycle phase', 'mitotic cell cycle', 'cell cycle process', 'M phase of mitotic cell cycle' and 'nuclear division', while the downregulated DEGs were primarily associated with 'response to wounding', 'anatomical structure morphogenesis', 'response to stimulus', 'response to chemical stimulus' and 'single-multi cellular organism process'. These results are in agreement with those of previously published studies suggesting that irregular and abnormal cell cycles or cell proliferation are closely associated with tumor proliferation and apoptosis (21-24), and that there is an association between repetitive wounding/stimuli and lung cancer (25).

The KEGG pathway analysis result revealed that upregulated DEGs were involved in 'Cell cycle', 'DNA replication', 'p53 signaling pathway', 'ECM-receptor interaction' and 'protein digestion and absorption'. Previous studies have 
Table II. Kyoto Encyclopedia of Genes and Genomes pathway analysis of DEGs associated with non-small cell lung cancer.

A, Upregulated DEGs

\begin{tabular}{|c|c|c|c|c|}
\hline Pathway ID & Name & Count & P-value & Genes \\
\hline hsa04110 & Cell cycle & 25 & $8.4 \times 10^{-11}$ & $\begin{array}{l}\text { CDK1, CDC6, E2F3, DBF4, TTK, ESPL1, CDC20, } \\
\text { CHEK1, MCM2, PTTG1, SFN, MCM4, CCNB1, } \\
\text { CCNE2, CCNE1, CDC45, CDKN2A, CCNB2, } \\
\text { MAD2L1, PLK1, PCNA, BUB1B, ORC6, ORC1, } \\
\text { CCNA2 }\end{array}$ \\
\hline hsa03030 & DNA replication & 10 & $8.8 \times 10^{-6}$ & $\begin{array}{l}\text { PRIM1, DNA2, RFC4, POLE2, PCNA, MCM2, } \\
\text { RNASEH2A, MCM4, FEN1, RPA3 }\end{array}$ \\
\hline hsa04115 & p53 signaling pathway & 13 & $1.2 \times 10^{-5}$ & $\begin{array}{l}\text { CDK1, CHEK1, SFN, PMAIP1, GTSE1, CCNB1, } \\
\text { CCNE2, CCNE1, CDKN2A, CCNB2, SERPINB5, } \\
\text { RRM2, IGFBP3 }\end{array}$ \\
\hline hsa04512 & $\begin{array}{l}\text { Extracellular matrix-receptor } \\
\text { interaction }\end{array}$ & 11 & $2.0 \times 10^{-3}$ & $\begin{array}{l}\text { IBSP, COMP, COL3A1, COL1A2, COL1A1, COL11A1, } \\
\text { THBS2, COL5A2, COL5A1, SPP1, HMMR }\end{array}$ \\
\hline hsa04974 & $\begin{array}{l}\text { Protein digestion and } \\
\text { absorption }\end{array}$ & 11 & $3.0 \times 10^{-3}$ & $\begin{array}{l}\text { KCNN4, COL17A1, COL7A1, PRSS2, COL3A1, } \\
\text { COL1A2, COL1A1, COL11A1, COL5A2, COL5A1, } \\
\text { COL10A1 }\end{array}$ \\
\hline
\end{tabular}

B, Downregulated DEGs

\begin{tabular}{|c|c|c|c|c|}
\hline Pathway ID & Name & Count & P-value & Genes \\
\hline hsa04610 & $\begin{array}{l}\text { Complement and coagulation } \\
\text { cascades }\end{array}$ & 16 & $4.2 \times 10^{-6}$ & $\begin{array}{l}\text { C7, C5AR1, C6, F8, SERPING1, C4BPA, C1QA, C8B, } \\
\text { C1QB, VWF, CD55, THBD, SERPIND1, CFD, CPB2, } \\
\text { PROS1 }\end{array}$ \\
\hline hsa05144 & Malaria & 12 & $5.9 \times 10^{-5}$ & $\begin{array}{l}\text { CSF3, GYPC, ICAM1, ITGAL, SELP, IL6, CD36, } \\
\text { PECAM1, ACKR1, TLR4, HBB, SELE }\end{array}$ \\
\hline hsa04514 & Cell adhesion molecules & 20 & $3.1 \times 10^{-4}$ & $\begin{array}{l}\text { ICAM1, ITGAL, SELP, CLDN18, OCLN, PTPRM, } \\
\text { CADM1, ICAM2, CLDN5, NECTIN3, HLA-DMA, } \\
\text { CDH5, SIGLEC1, CD34, ITGA8, PECAM1, ESAM, } \\
\text { JAM2, SELE, NEGR1 }\end{array}$ \\
\hline hsa04360 & Axon guidance & 18 & $6.3 \times 10^{-4}$ & $\begin{array}{l}\text { ABLIM1, PLXNA2, ABLIM3, EFNB2, NTN4, DPYSL2, } \\
\text { CXCL12, SLIT2, SLIT3, SEMA5A, SEMA6A, RND1, } \\
\text { SEMA6D, FYN, SEMA3G, CFL2, SEMA3E, ROBO2 }\end{array}$ \\
\hline hsa04924 & Renin secretion & 12 & $7.1 \times 10^{-4}$ & $\begin{array}{l}\text { AGTR1, ACE, ADRB2, ADRB1, PLCB4, PTGER4, } \\
\text { GUCY1A2, GUCY1A3, NPR1, AQP1, CACNA1D, } \\
\text { ITPR1 }\end{array}$ \\
\hline
\end{tabular}

p53, tumor protein 53; DEGs, differentially expressed genes.

indicated that the p53-independent structure-activity associations of mesogenic compounds are associated with cytotoxic effects $(26,27)$, and that disturbances in the p53 signaling pathway is associated with NSCLC (28). According to previous studies, ECM-receptor interactions were involved in cell adhesion (29), and it has been revealed that the ECM molecule hyaluronan induced focal adhesion, to signal the cytoskeletal changes required for the elevated cell motility observed in the processes of tumor cell progression, metastasis and invasion (30).

Notably, the downregulated DEGs were enriched in disease malaria during the KEGG enrichment analysis, which may suggest that anti-malaria compounds, artemisinin, dihydroartemisinin and artesunate, also have anticancer potential. The antimalarial drug, artemisinin, was previously used in the treatment of lung cancer, and Tong et al (31) identified that artemisinin inhibited tumor metastasis through $\mathrm{Wnt} / \beta$-catenin signaling. In addition, a previous study by Ashton et al (32) suggested that a commonly used anti-malarial drug, atovaquone, effectively increased the oxygen content inside cancer cells, therefore improving the efficiency of radiation treatment. Atovaquone rapidly decreased the oxygen consumption rate by $>80 \%$ in a range of cancer cell lines at pharmacological concentrations. In additional experiments, atovaquone killed 
Table III. Hub genes and rank of degrees ${ }^{\mathrm{a}}$.

Gene symbol

CDK1

PLK1

AURKB

CDC20

BIRC5

BUB1B

PCNA

CENPA

MAD2L1
Full name

Degree

Cyclin dependent kinase 1

Polo-like kinase 1

Aurora kinase B

46

Cell division cycle 20

42

Baculoviral initiator of apoptosis repeat containing $5 \quad 37$

BUB1 mitotic checkpoint serine/threonine kinase B 36

Proliferating cell nuclear antigen $\quad 35$

Centromere protein A $\quad 34$

MAD2 mitotic arrest deficient-like 1
A
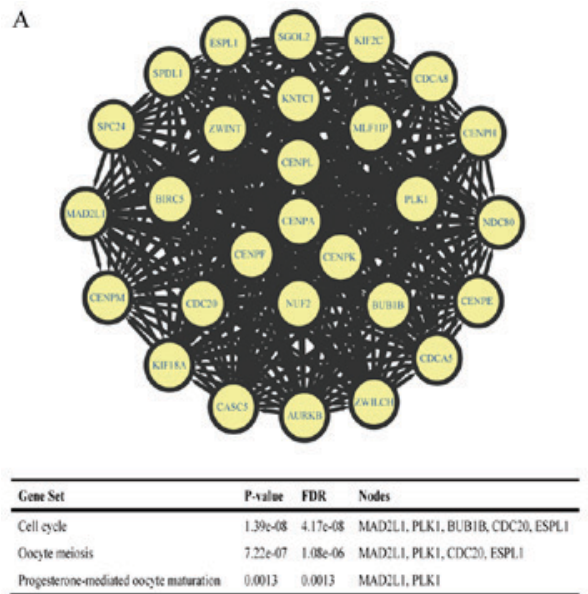

C

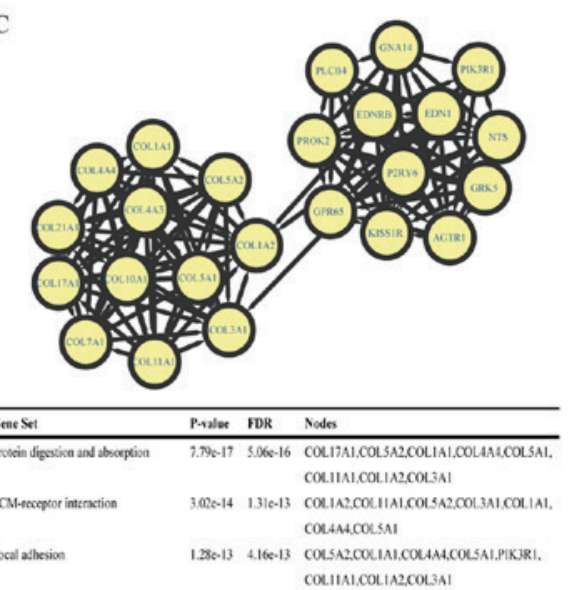

B

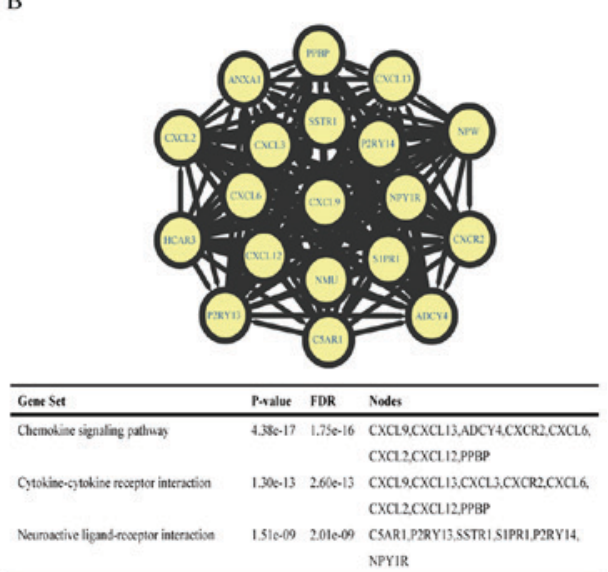

D
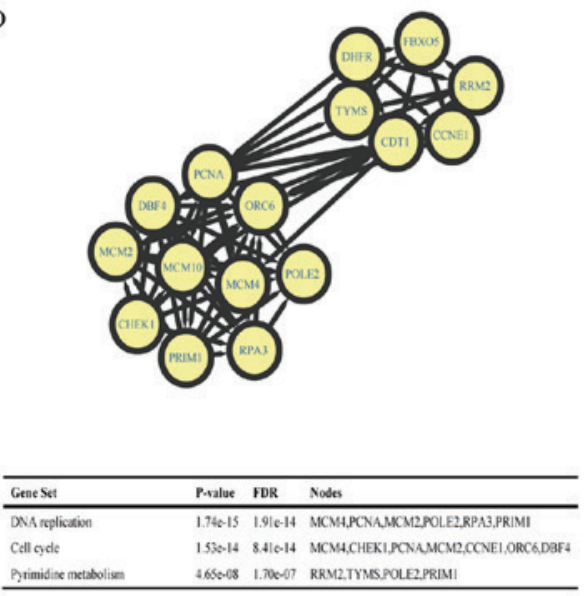

$\mathrm{E}$

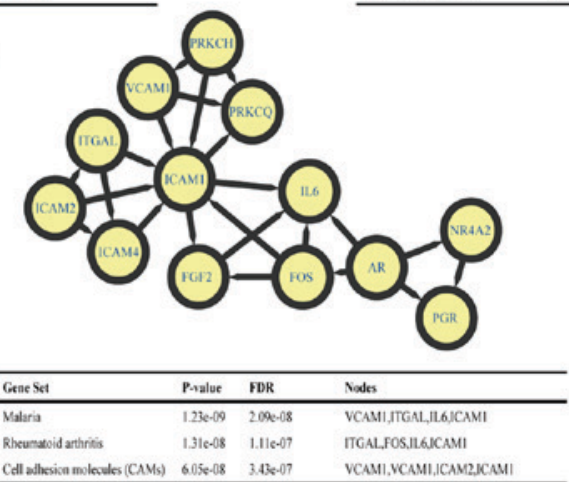

Figure 3. Top 5 modules from the high-score protein-protein interactive network. (A-E) Modules 1-5 and their enriched pathways. FDR, false discovery rate. 


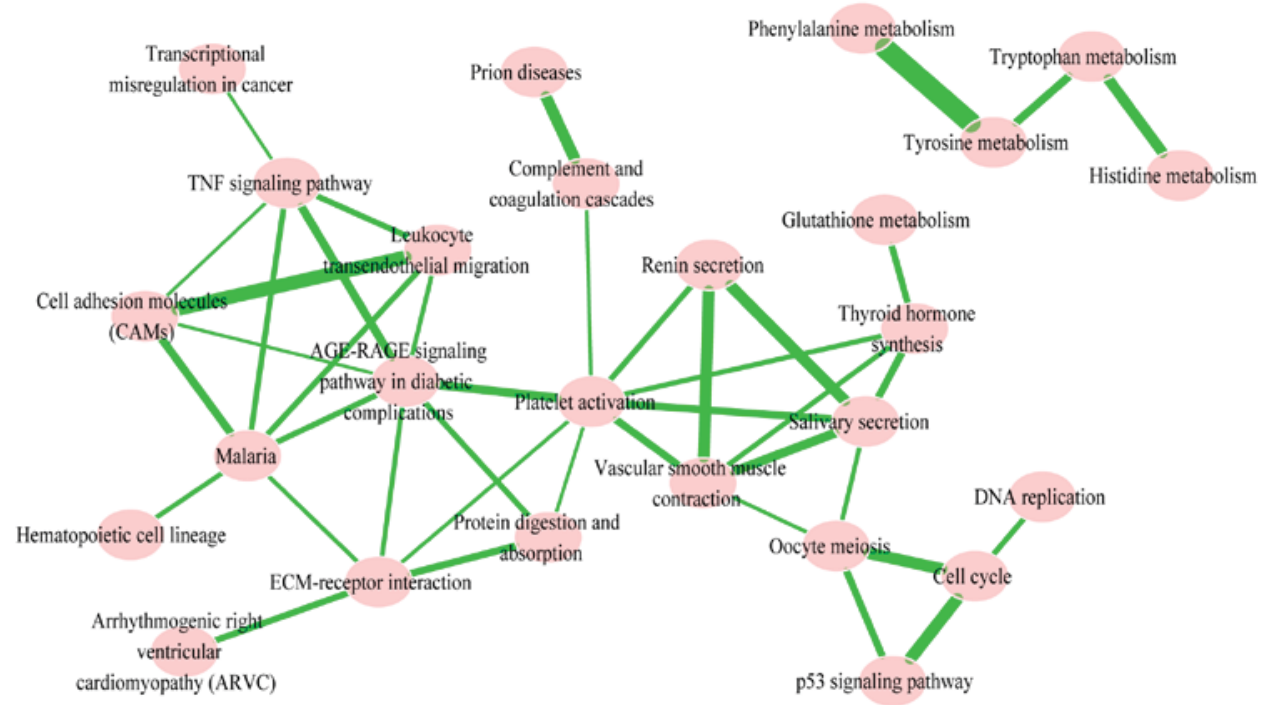

Figure 4. Pathway crosstalk among differentially expressed genes-enriched pathways. AGE-RAGE, advanced glycation endproducts-receptor for AGE; ECM, extracellular matrix; p53, tumor protein 53; TNF, tumor necrosis factor.

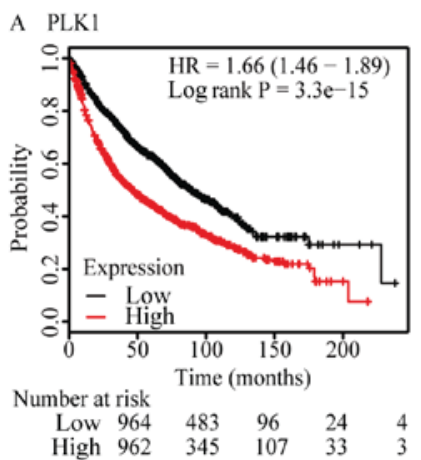

D BIRC5
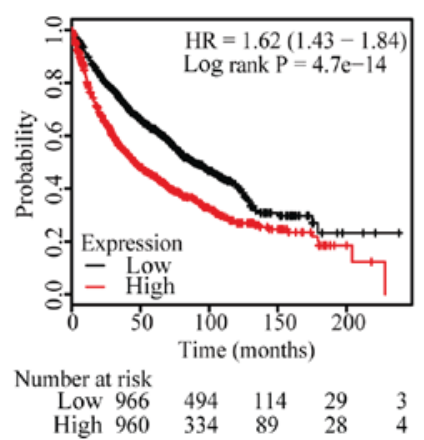

G MAD2L1

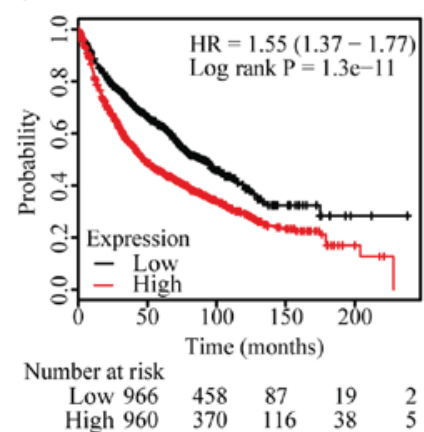

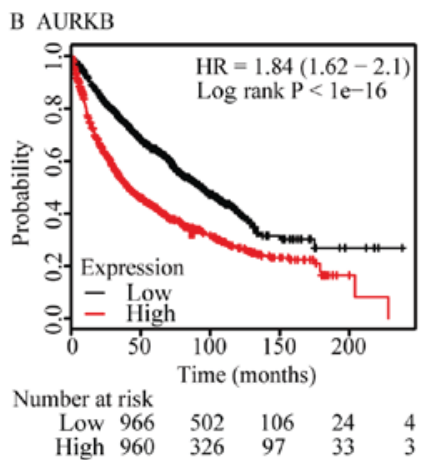

E BUBIB

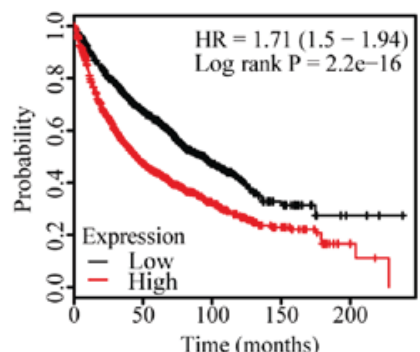

Number at risk

$\begin{array}{lllll}\text { Low } 963 & 483 & 103 & 23 & 3\end{array}$ High $963 \quad 345 \quad 100 \quad 34 \quad 4$

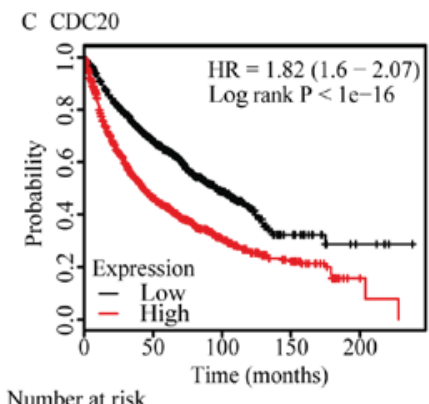

Number at risk

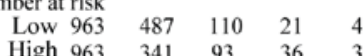

F CENPA

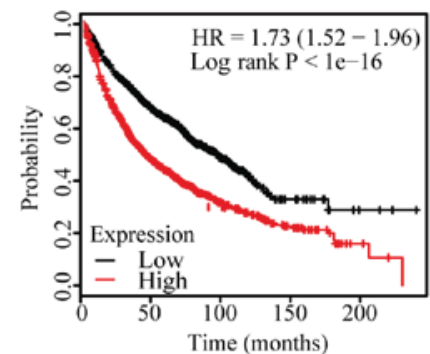

Number at risk

Low $963 \quad 485 \quad 101 \quad 20 \quad 3$

High $963 \quad 343 \quad 102 \quad 37 \quad 4$

H PCNA

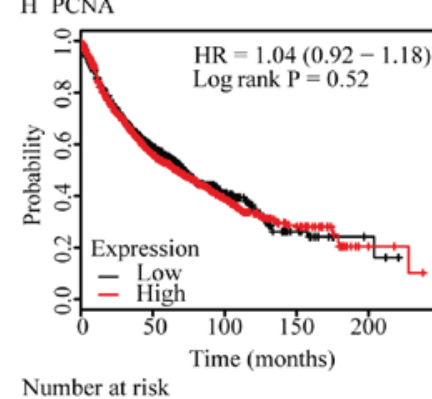

$$
\begin{array}{ccccc}
\text { Number at risk } & & & & \\
\text { Low 963 } & 392 & 84 & 17 & 3 \\
\text { High 963 } & 436 & 119 & 40 & 4
\end{array}
$$

Figure 5. Survival analysis of hub genes. (A) PLK, (B) AURKB, (C) CDC20, (D) BIRC5, (E) BUB1B, (F) CENPA and (G) MAD2L1 expression indicated significantly lower survival rates, compared with low expression samples. (H) PCNA expression did not exhibit a significantly different survival rates. PLK, polo-like kinase 1; AURKB, aurora kinase B; CDC20, cell division cycle 20; BIRC5, baculoviral initiator of apoptosis repeat containing 5; BUB1B, BUB1 mitotic checkpoint serine/threonine kinase B; CENPA, centromere protein A; MAD2L1, MAD2 mitotic arrest deficient-like 1; PCNA, proliferating cell nuclear antigen. 
$90 \%$ of cancer cells in an in vitro lung cancer tumor model involving radiotherapy (32).

By constructing a PPI network with DEGs, the present study identified the top degree hub genes: CDK1, PLK1, AURKB, CDC20, BIRC5, BUB1B, PCNA, CENPA and MAD2L1. CDK1 exhibited the highest degree of connectivity among these hub genes. CDK1, a protein-coding gene, serves a key role in the control of the eukaryotic cell cycle by modulating the centrosome cycle and mitotic onset, promoting G2-M transition and regulating G1 progress and G1-S transition via association with multiple interphase cyclins (33). CDK1 is an adverse prognostic biomarker of lung adenocarcinoma (LUAD), and increased expression of CDK1 was revealed to be associated with a higher risk of cancer recurrence and poor survival, compared with the normal expression of CDK1 in patients with LUAD (34). Danilov et al (35) also demonstrated that using dinaciclib to inhibit the expression of CDK1 induced anaphase catastrophe in lung cance (35). The second hub gene, PLK1, performs several important functions throughout the $\mathrm{M}$ phase of the cell cycle, including the regulation of centrosome maturation and spindle assembly, the removal of cohesins from chromosome arms, the inactivation of anaphase-promoting complex/cyclosome (APC/C) inhibitors and the regulation of mitotic exit and cytokinesis (36). A previous study demonstrated that PLK1 may promote tumor cell survival by regulating Myc stabilization; inhibitors of PLK1 preferentially induced potent apoptosis level in MYCN-amplified tumor cells from neuroblastoma and small cell lung cancer, and synergistically potentiated the therapeutic efficacies of Bcl-2 antagonists (37). AURKB, the third hub gene, encodes a member of the aurora kinase subfamily of serine/threonine kinases, and participates in the regulation of alignment and segregation of chromosomes during mitosis and meiosis (38). A previous study has confirmed the antitumor and radiosensitizing activities of daurinol in human lung cancer cells through the inhibition of AURKB (39). In addition, CDC20 serves as a regulatory protein interacting with several other proteins at multiple points in the cell cycle (40). A previous study suggested that CDC20 is a critical regulator in glioma tumors, initiating cell proliferation and survival (41). High levels of CDC20 expression are a key component of the spindle assembly checkpoint; CDC20 has been identified in various malignancies and serves a vital role in tumorigenesis and progression (42-44). BIRC5, the member of the IAP gene family, encodes negative regulatory proteins that prevent apoptotic cell death. Han et al (45) suggested that the upregulation of the anti-apoptosis gene BIRC5 will lead to the inhibition of p53 signaling in H1650GR cells. BUB1B serves a role in the inhibition of the $\mathrm{APC} / \mathrm{C}$, delaying the onset of anaphase and ensuring proper chromosome segregation (46). Chen et al (47) also revealed that BUB1B may serve as target gene in lung carcinoma as a result of PPI network analysis. In addition, high levels of BUB1B expression are associated with disease progression and poor survival in patients with lung adenocarcinoma (47). PCNA acts as a homotrimer and assists in increasing the processivity of leading strand synthesis during DNA replication (48). Bodduluru et al (49) revealed that benzo[a] pyrene may induce pulmonary carcinogenesis by modulating PCNA expression. CENPA encodes a centromere protein that contains a histone $\mathrm{H} 3$-associated histone fold domain required for targeting to the centromere (50). This domain is one of the basic components of the human active kinetochore, which serves an important role in cell-cycle regulation, cell survival and genetic stability (51). Toh et al (52) identified that CENPA may be considered as a prospective diagnostic and prognostic biomarker of lung adenocarcinoma. MAD2L1 is a component of the mitotic spindle assembly checkpoint that prevents the onset of anaphase until all chromosomes are properly aligned at the metaphase plate (53). As aforementioned, MAD2L1 is closely associated with the carcinogenesis of lung adenocarcinoma (34). Guo et al (54) performed a case-control analysis, indicating an association between the concentration of MAD2L1 Leu84Met SNP gene product and the risk of lung cancer in an allele dose-dependent manner, with the result demonstrating that the expression level of MAD2L1 Leu84Met SNP was linearly associated with the risk of lung cancer.

Considering the enrichment results of the top 5 modules from the PPI network genes in the present study, it was demonstrated that NSCLC was associated with 'cell cycle', 'chemokine signaling pathway', 'protein digestion and absorption', 'DNA replication' and 'malaria'.

In the chemokine signaling pathway (hsa04062; KEGG database), chemokines are a type of small chemoattractant peptide, which may provide directional cues for cell trafficking; this is the key for the protective host response (55). In addition, chemokines regulate a plethora of biological processes in hematopoietic cells that lead to cellular activation, differentiation and survival $(55,56)$. A previous study has revealed that chemokines are vital in the pathogenesis of NSCLC and NSCLC cells are rich in the secreted protein CXCL12 (57). Another study has suggested that the methylation of CXCL12 has a marked correlation with NSCLC prognosis (58), and that CXCL12-mediated adhesion and survival signals are associated with chemo-resistance in lung cancer (59).

During the survival analysis of the present study, KMplot was used to assess the effect of high expression levels of the hub genes in patients with lung cancer. There were 8 genes available in the database, with only CDK1 not matching HGU133A and HGU133Aplus2 probe set IDs in the KMplot database. Notably, 7 of the 8 genes indicated significantly low survival rates, compared with low expression samples $(\mathrm{P}<0.05)$; the remaining gene, PCNA, did not. In addition, a number of previous studies have analyzed the association between the expression of PCNA and NSCLC postoperative survival time and did not identify a significant correlation (60-63). This may be due to the fact that the prognosis of lung cancer may be affected by a variety of factors, including pathological type and stage, differentiation, treatment, complications, age, physical condition and the expression of PCNA $(60,64-67)$. It is difficult to predict the prognosis of lung cancer by considering the effecters of PCNA alone.

In conclusion, the results from the present study provided a wider analysis of the DEGs associated with NSCLC, and identified certain key pathways in the progress of NSCLC, which may provide guidance for future studies. Nevertheless, a number of biomarkers associated with NSCLC remain uncharacterized; additional biological and bioinformatics analyses are required. 


\section{Acknowledgements}

The authors would like to thank Dr Yanchao Mu (Clinical Laboratory of Anyang Children's Hospital, Henan, China) for their technical guidance.

\section{Funding}

No funding was received.

\section{Availability of data and materials}

Not applicable.

\section{Authors' contributions}

QT and HZ wrote the main part of the manuscript and took part in the planning and execution of the experiments. MK and XM took part in the development of the analysis code, planned and carried out the main part of the experiments. XC provided language guidance designed this experiment and revised the manuscript. All authors have read and approved the final manuscript.

\section{Ethics approval and consent to participate}

Not applicable.

\section{Consent for publication}

Not applicable.

\section{Competing interests}

The authors declare that they have no competing interests.

\section{References}

1. Siegel RL, Miller KD and Jemal A: Cancer statistics, 2015. CA Cancer J Clin 65: 5-29, 2015.

2. Spira A and Ettinger DS: Multidisciplinary management of lung cancer. N Engl J Med 350: 379-392, 2004.

3. Ettinger DS, Akerley W, Borghaei H, Chang AC, Cheney RT, Chirieac LR, D'Amico TA, Demmy TL, Ganti AK, Govindan R, et al: Non-small cell lung cancer. J Natl Compr Canc Netw 10: 1236-1271, 2012.

4. Bejjani BA and Shaffer LG: Clinical utility of contemporary molecular cytogenetics. Annu Rev Genomics Hum Genet 9: 71-86, 2008.

5. Zhang C, Li HR, Fan JB, Wang-Rodriguez J, Downs T, Fu XD and Zhang MQ: Profiling alternatively spliced mRNA isoforms for prostate cancer classification. BMC Bioinformatics 7: 202, 2006.

6. Zhu M, Deng X,Joshi T, Xu D, Stacey G and Cheng J: Reconstructing differentially co-expressed gene modules and regulatory networks of soybean cells. BMC Genomics 13: 437, 2012.

7. Denoix PF: Enquete permanent dans les centres anticancereaux, Bull Inst Nat Hyg 1:70-75, 1946 (In French).

8. Meister M, Belousov A, Xu EC, et al: Intra-tumor heterogeneity of gene expression profiles in early stage non-small cell lung cancer. J Bioinf Res Stud 1:1, 2014.

9. Gautier L, Cope L, Bolstad BM and Irizarry RA: Affy-analysis of Affymetrix GeneChip data at the probe level. Bioinformatics 20: 307-315, 2004.

10. Phipson B, Lee S, Majewski IJ, Alexander WS and Smyth GK: Robust hyperparameter estimation protects against hypervariable genes and improves power to detect differential expression. Ann Appl Stat 10: 946-963, 2016.
11. Martucci D, Masseroli M and Pinciroli F: Gene ontology application to genomic functional annotation, statistical analysis and knowledge mining. Stud Health Technol Inform 102: 108-131, 2004.

12. Kanehisa M: The KEGG database. Novartis Found Symp 247: 91-103, 119-128, 244-152, 2002.

13. Dennis G Jr, Sherman BT, Hosack DA, Yang J, Gao W, Lane HC and Lempicki RA: DAVID: Database for annotation, visualization, and integrated discovery. Genome Biol 4: P3, 2003.

14. Shannon P, Markiel A, Ozier O, Baliga NS, Wang JT, Ramage D, Amin N, Schwikowski B and Ideker T: Cytoscape: A software environment for integrated models of biomolecular interaction networks. Genome Res 13: 2498-2504, 2003.

15. Langley SR, Dwyer J, Drozdov I, Yin X and Mayr M: Proteomics: From single molecules to biological pathways. Cardiovasc Res 97: 612-622, 2013.

16. Burrell RA, McGranahan N, Bartek J and Swanton C: The causes and consequences of genetic heterogeneity in cancer evolution. Nature 501: 338-345, 2013.

17. Liu M, Fan R, Liu X, Cheng F and Wang J: Pathways and networks-based analysis of candidate genes associated with nicotine addiction. PLoS One 10: e0127438, 2015.

18. Szasz AM, Lanczky A, Nagy A, Förster S, Hark K, Green JE, Boussioutas A, Busuttil R, Szabó A and Győrffy B: Cross-validation of survival associated biomarkers in gastric cancer using transcriptomic data of 1,065 patients. Oncotarget 7: 49322-49333, 2016.

19. Ramalingam SS, Owonikoko TK and Khuri FR: Lung cancer: New biological insights and recent therapeutic advances. CA Cancer J Clin 61: 91-112, 2011.

20. Gridelli C, Rossi A, Carbone DP, Guarize J, Karachaliou N, Mok T, Petrella F, Spaggiari L and Rosell R: Non-small-cell lung cancer. Nat Rev Dis Primers 1: 15009, 2015.

21. An Q, Han C, Zhou Y, Li F, Li D, Zhang X, Yu Z, Duan Z and Kan Q: Matrine induces cell cycle arrest and apoptosis with recovery of the expression of miR-126 in the A549 non-small cell lung cancer cell line. Mol Med Rep 14: 4042-4048, 2016.

22. Jung CK, Jung JH, Lee KY, Kang CS, Kim M, Ko YH and Oh CS: Centrosome abnormalities in non-small cell lung cancer: correlations with DNA aneuploidy and expression of cell cycle regulatory proteins. Pathol Res Pract 203: 839-847, 2007.

23. Rao PC, Begum S, Jahromi MA, Jahromi ZH, Sriram S and Sahai M: Cytotoxicity of withasteroids: Withametelin induces cell cycle arrest at $\mathrm{G} 2 / \mathrm{M}$ phase and mitochondria-mediated apoptosis in non-small cell lung cancer A549 cells. Tumour Biol 37: 12579-12587, 2016.

24. Wang L, Xu J, Zhao C, Zhao L and Feng B: Antiproliferative, cell-cycle dysregulation effects of novel asiatic acid derivatives on human non-small cell lung cancer cells. Chem Pharm Bull (Tokyo) 61: 1015-1023, 2013.

25. Haura EB: Is repetitive wounding and bone marrow-derived stem cell mediated-repair an etiology of lung cancer development and dissemination? Med Hypotheses 67: 951-956, 2006.

26. Hai J, Sakashita S, Allo G, Ludkovski O, Ng C, Shepherd FA and Tsao MS: Inhibiting MDM2-p53 interaction suppresses tumor growth in patient-derived non-small cell lung cancer xenograft models. J Thorac Oncol 10: 1172-1180, 2015.

27. In JK, Kim JK, Oh JS and Seo DW: 5-Caffeoylquinic acid inhibits invasion of non-small cell lung cancer cells through the inactivation of p70S6K and Akt activity: Involvement of p53 in differential regulation of signaling pathways. Int J Oncol 48: 1907-1912, 2016.

28. Fukushi S, Yoshino H, Yoshizawa A and Kashiwakura I: p53-independent structure-activity relationships of 3-ring mesogenic compounds' activity as cytotoxic effects against human non-small cell lung cancer lines. BMC Cancer 16: 521, 2016.

29. Albelda SM and Buck CA: Integrins and other cell adhesion molecules. FASEB J 4: 2868-2880, 1990.

30. Hall CL and Turley EA: Hyaluronan: RHAMM mediated cell locomotion and signaling in tumorigenesis. J Neurooncol 26: 221-229, 1995.

31. Tong Y, Liu Y, Zheng H, Zheng L, Liu W, Wu J, Ou R, Zhang G, $\mathrm{Li} \mathrm{F}, \mathrm{Hu} \mathrm{M}$, et al: Artemisinin and its derivatives can significantly inhibit lung tumorigenesis and tumor metastasis through Wnt/ $\beta$-catenin signaling. Oncotarget 7: 31413-31428, 2016.

32. Ashton TM, Fokas E, Kunz-Schughart LA, Folkes LK, Anbalagan S, Huether M, Kelly CJ, Pirovano G, Buffa FM, Hammond EM, et al: The anti-malarial atovaquone increases radiosensitivity by alleviating tumour hypoxia. Nature Commun 7: 12308, 2016. 
33. Castedo M, Perfettini JL, Roumier T and Kroemer G: Cyclin-dependent kinase-1: Linking apoptosis to cell cycle and mitotic catastrophe. Cell Death Differ 9: 1287-1293, 2002.

34. Shi YX, Zhu T, Zou T, Zhuo W, Chen YX, Huang MS, Zheng W, Wang CJ, Li X, Mao XY, et al: Prognostic and predictive values of CDK1 and MAD2L1 in lung adenocarcinoma. Oncotarget 7: 85235-85243, 2016.

35. Danilov AV, Hu S, Orr B, Godek K, Mustachio LM, Sekula D, Liu X, Kawakami M, Johnson FM, Compton DA, et al: Dinaciclib induces anaphase catastrophe in lung cancer cells via inhibition of cyclin-dependent kinases 1 and 2. Mol Cancer Ther 15: 2758-2766, 2016

36. Lane HA and Nigg EA: Antibody microinjection reveals an essential role for human polo-like kinase 1 (Plk1) in the functional maturation of mitotic centrosomes. J Cell Biol 135: 1701-1713, 1996.

37. Xiao D, Yue M, Su H, Ren P, Jiang J, Li F, Hu Y, Du H, Liu H and Qing G: Polo-like kinase-1 regulates Myc stabilization and activates a feedforward circuit promoting tumor cell survival Mol Cell 64: 493-506, 2016.

38. Goldenson B and Crispino JD: The aurora kinases in cell cycle and leukemia. Oncogene 34: 537-545, 2015.

39. Woo JK, Kang JH, Shin D, Park SH, Kang K, Nho CW, Seong JK, Lee SJ and Oh SH: Daurinol enhances the efficacy of radiotherapy in lung cancer via suppression of aurora kinase $a / b$ expression. Mol Cancer Ther 14: 1693-1704, 2015.

40. Weinstein J, Jacobsen FW, Hsu-Chen J, Wu T and Baum LG: A novel mammalian protein, $\mathrm{p} 55 \mathrm{CDC}$, present in dividing cells is associated with protein kinase activity and has homology to the Saccharomyces cerevisiae cell division cycle proteins Cdc20 and Cdc4. Mol Cell Biol 14: 3350-3363, 1994.

41. Xie Q, Wu Q, Mack SC, Yang K, Kim L, Hubert CG, Flavahan WA, Chu C, Bao S and Rich JN: CDC20 maintains tumor initiating cells. Oncotarget 6: 13241-13254, 2015.

42. Choi JW, Kim Y, Lee JH and Kim YS: High expression of spindle assembly checkpoint proteins CDC20 and MAD2 is associated with poor prognosis in urothelial bladder cancer. Virchows Arch 463: 681-687, 2013

43. Chang DZ, Ma Y, Ji B, Liu Y, Hwu P, Abbruzzese JL, Logsdon C and Wang H: Increased CDC20 expression is associated with pancreatic ductal adenocarcinoma differentiation and progression. J Hematol Oncol 5: 15, 2012.

44. Kato T, Daigo Y, Aragaki M, Ishikawa K, Sato M and Kaji M: Overexpression of CDC20 predicts poor prognosis in primary non-small cell lung cancer patients. J Surg Oncol 106: 423-430, 2012.

45. Han X, Liu M, Wang S, Lv G, Ma L, Zeng C and Shi Y: An integrative analysis of the putative gefitinib-resistance related genes in a lung cancer cell line model system. Curr Cancer Drug Targets 15: 423-434, 2015.

46. Davenport JW, Fernandes ER, Harris LD, Neale GA and Goorha R: The mouse mitotic checkpoint gene bublb, a novel bub1 family member, is expressed in a cell cycle-dependent manner. Genomics 55: 113-117, 1999.

47. Chen H, Lee J, Kljavin NM, Haley B, Daemen A, Johnson L and Liang Y: Requirement for BUB1B/BUBR1 in tumor progression of lung adenocarcinoma. Genes Cancer 6: 106-118, 2015.

48. Moldovan GL, Pfander B and Jentsch S: PCNA, the maestro of the replication fork. Cell 129: 665-679, 2007

49. Bodduluru LN, Kasala ER, Madhana RM, Barua CC, Hussain MI, Haloi P and Borah P: Naringenin ameliorates inflammation and cell proliferation in benzo(a)pyrene induced pulmonary carcinogenesis by modulating CYP1A1, NFkappaB and PCNA expression. Int Immunopharmacol 30: 102-110, 2016.

50. Chueh AC, Wong LH, Wong N and Choo KH: Variable and hierarchical size distribution of L1-retroelement-enriched CENP-A clusters within a functional human neocentromere. Hum Mol Genet 14: 85-93, 2005.
51. Folco HD,Pidoux AL, UranoT and Allshire RC: Heterochromatin and RNAi are required to establish CENP-A chromatin at centromeres. Science 319: 94-97, 2008.

52. Toh SH, Prathipati P, Motakis E, Kwoh CK, Yenamandra SP and Kuznetsov VA: A robust tool for discriminative analysis and feature selection in paired samples impacts the identification of the genes essential for reprogramming lung tissue to adenocarcinoma. BMC Genomics 12 (Suppl 3): S24, 2011

53. Xu L, Deng HX, Yang Y, Xia JH, Hung WY and Siddque T: Assignment of mitotic arrest deficient protein 2 (MAD2L1) to human chromosome band $5 \mathrm{q} 23.3$ by in situ hybridization. Cytogenet Cell Genet 78: 63-64, 1997.

54. Guo Y, Zhang X, Yang M, Miao X, Shi Y, Yao J, Tan W, Sun T, Zhao D, Yu D, et al: Functional evaluation of missense variations in the human MAD1L1 and MAD2L1 genes and their impact on susceptibility to lung cancer. J Med Genet 47: 616-622, 2010.

55. Zlotnik A, Burkhardt AM and Homey B: Homeostatic chemokine receptors and organ-specific metastasis. Nat Rev Immunol 11: 597-606, 2011

56. Zlotnik A and Yoshie O: The chemokine superfamily revisited. Immunity 36: 705-716, 2012.

57. Wald O, Shapira OM and Izhar U: CXCR4/CXCL12 axis in non small cell lung cancer (NSCLC) pathologic roles and therapeutic potential. Theranostics 3: 26-33, 2013 .

58. Suzuki M, Mohamed S, Nakajima T, Kubo R, Tian L, Fujiwara T, Suzuki H, Nagato K, Chiyo M, Motohashi S, et al: Aberrant methylation of CXCL12 in non-small cell lung cancer is associated with an unfavorable prognosis. Int J Oncol 33: 113-119, 2008.

59. Hartmann TN, Burger JA, Glodek A, Fujii N and Burger M: CXCR4 chemokine receptor and integrin signaling co-operate in mediating adhesion and chemoresistance in small cell lung cancer (SCLC) cells. Oncogene 24: 4462-4471, 2005.

60. Ebina M, Steinberg SM, Mulshine JL and Linnoila RI: Relationship of p53 overexpression and up-regulation of proliferating cell nuclear antigen with the clinical course of non-small cell lung cancer. Cancer Res 54: 2496-2503, 1994.

61. Matturri L, Lavezzi AM, Grignani F, Salomoni G and Roviaro GC: The prognostic value of cell proliferation in non-small cell lung cancer assessed with tritiated thymidine and anti-PCNA antibodies. Eur J Cancer 30A: 1397-1398, 1994.

62. Alemany Monraval P, Martorell Cebollada M, Salvador Villalba I and Martínez Leandro E: Study of the expression of proliferating cell nuclear antigen and p185 in non-small cell lung carcinoma. Arch Bronconeumol 32 (In Spanish): 165-169, 1996.

63. Castellano VM, Sotelo T, Ballestin C, Lopez-Encuentra A and Varela G: Analysis of proliferating cell nuclear antigen (PCNA) expression in 24 cases of primary non-small cell pulmonary carcinomas and correlation with survival. Arch Bronconeumol 32 (In Spanish): 127-131, 1996

64. Guo X, Li D, Wu Y, Chen Y, Zhou X, Wang X, Huang X, Li X, Yang $\mathrm{H}$ and $\mathrm{Xing}$ J: Genetic variants in genes of tricarboxylic acid cycle key enzymes are associated with prognosis of patients with non-small cell lung cancer. Lung Cancer 87: 162-168, 2015.

65. Di JZ, Peng JY and Wang ZG: Prevalence, clinicopathological characteristics, treatment, and prognosis of intestinal metastasis of primary lung cancer: A comprehensive review. Surg Oncol 23: 72-80, 2014.

66. North CM and Christiani DC: Women and lung cancer: What is new? Semin Thorac Cardiovasc Surg 25: 87-94, 2013.

67. Lin J and Beer DG: Molecular predictors of prognosis in lung cancer. Ann Surg Oncol 19: 669-676, 2012.

This work is licensed under a Creative Commons Attribution-NonCommercial-NoDerivatives 4.0 International (CC BY-NC-ND 4.0) License. 